\title{
TPX2 promotes glioma cell proliferation and invasion via activation of the AKT signaling pathway
}

\author{
JIAN-JUN GU ${ }^{1,2^{*}}$, JIAN-HE ZHANG ${ }^{1}$, HONG-JIE CHEN ${ }^{1 *}$ and SHOU-SEN WANG ${ }^{1}$ \\ ${ }^{1}$ Department of Neurosurgery, Fuzhou General Hospital, Xiamen University Medical College, Fuzhou, Fujian 350025; \\ ${ }^{2}$ Department of Neurosurgery, Taizhou People's Hospital Affiliated to Medical College of Nantong University, \\ Taizhou, Jiangsu 225300, P.R. China
}

Received June 13, 2015; Accepted October 14, 2016

DOI: $10.3892 / \mathrm{ol} .2016 .5371$

\begin{abstract}
Glioblastoma multiforme (GBM) is the most common and most malignant type of primary adult brain cancer. The most common phenotype associated with GBM is cellular invasion; however, the molecular mechanisms governing this process are poorly understood. Targeting protein for Xenopus kinesin-like protein 2 (TPX2) is a nuclear protein with roles in cellular proliferation and mitotic spindle assembly. TPX2 is overexpressed in various malignancies, including human malignant astrocytoma. Despite this finding, the exact role of TPX2 in human glioma is not well defined. The present study reports the elevated expression of TPX2 in a number of glioma cell lines. TPX 2 overexpression promoted cellular proliferation, decreased the percentage of cells in G0/G1 phase, and increased invasion of both U251 and U87 cells. Overexpression of TPX2 also significantly enhanced the phosphorylation of AKT, decreased the expression of $\mathrm{p} 21$, and increased the expression of cyclin D1 and matrix metallopeptidase (MMP)-9. In both U251 and U87 cells, knockdown of TPX 2 resulted in phenotypes that are in direct contrast to those observed following TPX2 overexpression. Specifically, TPX2 knockdown inhibited cell proliferation, increased the percentage of cells in G0/G1 phase, inhibited invasion, decreased AKT phosphorylation, decreased the expression of MMP-9 and cyclin D1, and increased p21 expression. The AKT inhibitor IV in large part phenocopied the effect of TPX2 knockdown. The present data suggest that TPX2 promotes glioma cell proliferation and invasion via AKT signaling.
\end{abstract}

Correspondence to: Dr Shou-Sen Wang, Department of Neurosurgery, Fuzhou General Hospital, Xiamen University Medical College, 156 Road Xi'erhuanbei, Fuzhou, Fujian 350025, P.R. China

E-mail:wshsen@126.com

*Contributed equally

Key words: glioma, TPX2, AKT, proliferation, invasion

\section{Introduction}

Glioblastoma multiforme (GBM) is the most malignant type of brain cancer affecting the central nervous system. Patients with GBM have a mean survival of only 14.6 months (1). Standard therapies for glioma, including surgery, radiation and chemotherapy, are only effective in treating high-grade tumors. The cancer's pathogenesis is not very well defined, but invasive growth is a known hallmark of GBM; this is also a major reason why GBM is associated with such poor prognosis (2). Therefore, an increased understanding of the biological and molecular nature of GBM is required.

Targeting protein for Xenopus kinesin-like protein 2 (TPX2) is a cell cycle-regulated nuclear protein with roles in proliferation and mitotic spindle assembly $(3,4)$. TPX2 overexpression induces centrosome amplification and leads to DNA polyploidy (5). Along these lines, TPX2 has been implicated as an oncogene in several human cancers, including lung cancer, cervical cancer, bladder cancer and human malignant astrocytoma (6-9). A number of studies have established that activated AKT signaling upregulates the expression of matrix metallopeptidase (MMP)-2 and MMP-9 $(10,11)$. TPX2 knockdown has been shown to suppress the proliferation and invasion of hepatocellular carcinoma cells via inactivation of AKT signaling and inhibition of the expression of MMP-2 and MMP-9 (12). Li et al also reported that TPX2 knockdown inhibited cell proliferation and increased early-stage apoptosis in glioma U87 cells (9). TPX2 knockdown was also shown to decrease the levels of Aurora A, Ras-related nuclear protein (Ran) and cyclin B1, and to increase the expression of c-Myc and p53 (9).

In the present study, increased expression of TPX2 was detected in multiple glioma cell lines. Additionally, TPX2 knockdown was able to inhibit glioma cell proliferation and invasion via inactivation of the AKT pathway. It was also observed that a small molecule inhibitor of AKT phenocopied the effects of TPX2 knockdown. Our results demonstrate that TPX2 knockdown suppresses cell proliferation and invasion via inactivation of AKT signaling in glioma cells.

\section{Materials and methods}

Cell culture and transfection. Normal human astrocytes (NHAs) were purchased from ScienCell Research Laboratories (Carlsbad, 
CA, USA). The glioma cell lines U251, U87 and LN229 were obtained from the Institute of Biochemistry and Cell Biology (Shanghai Institutes for Biological Sciences, Chinese Academy of Sciences, Shanghai, China). Cells were grown in Dulbecco's modified Eagle's medium (DMEM; Invitrogen; Thermo Fisher Scientific, Inc., Waltham, MA, USA) supplemented with $10 \%$ fetal bovine serum (FBS; Sigma-Aldrich; Merck Millipore, Darmstadt, Germany) and $1 \%$ penicillin/streptomycin at $37^{\circ} \mathrm{C}$ in an atmosphere of $5 \% \mathrm{CO}_{2}$. Specific small interfering RNA (siRNA) targeting TPX2 (sc-37653) and control siRNA (sc-37007) were purchased from Santa Cruz Biotechnology, Inc. (Dallas, TX, USA). Glioma cells were divided into three groups, including the mock, control siRNA and TPX2 siRNA groups. Glioma cells were seeded at a concentration of $2 \times 10^{5}$ cells/well in a 6-well plate (Corning Inc., Corning, NY, USA) and cultured overnight. Cells were then transfected with $100 \mathrm{nM}$ siRNA using Lipofectamine 2000 (Invitrogen; Thermo Fisher Scientific, Inc.) according to the manufacturer's protocol. The mock cells were treated with Lipofectamine 2000 only. The transfection medium was replaced with complete medium at $24 \mathrm{~h}$ post-transfection, and cells were incubated at $37^{\circ} \mathrm{C}$ in a $5 \% \mathrm{CO}_{2}$ atmosphere. AKT inhibitor IV was purchased from Calbiochem (EMD Millipore, Billerica, MA, USA).

Construction of the eukaryotic expression vector pcDNA-TPX2. TPX2 full-length cDNA was amplified by polymerase chain reaction (PCR) using the following TPX2-specific primers: 5'-CGGGATCCATGTCACAAG TTAAAAGCTC-3' (forward) and 5'-GCTCTAGATTAGCAG TGGAATCGAGTGGAG-3' (reverse). The cycling conditions were as follows: $94^{\circ} \mathrm{C}$ for $4 \mathrm{~min} ; 30$ cycles at $94^{\circ} \mathrm{C}$ for $90 \mathrm{sec}$, $65^{\circ} \mathrm{C}$ for $30 \mathrm{sec}$ and $72^{\circ} \mathrm{C}$ for $1 \mathrm{~min}$; and $72^{\circ} \mathrm{C}$ for $5 \mathrm{~min}$. The TPX2 PCR product and empty vector pcDNA3.1 (Thermo Fisher Scientific, Inc.) were digested with EcoRI and BamHI. pcDNA-TPX2 was generated by subcloning TPX2 into pcDNA3.1 using T4 DNA ligase (Thermo Fisher Scientific, Inc.). Glioma cells were divided into three groups, including the mock, pcDNA3.1 and pcDNA-TPX2 groups. Glioma cells were seeded at a concentration of $2 \times 10^{5}$ cells/well in a 6-well plate and cultured overnight. Cells were then transfected with pcDNA-TPX2 or TPX2-siRNA $(10 \mu \mathrm{l})$ in $250 \mu \mathrm{l}$ Opti-MEM I reduced serum medium (Thermo Fisher Scientific, Inc.) using Lipofectamine 2000. The mock cells were treated with Lipofectamine 2000 only.

$R N A$ extraction and reverse transcription-quantitative $P C R$ $(R T-q P C R)$. Total RNA was extracted from cultured cells using TRIzol reagent (Invitrogen; Thermo Fisher Scientific, Inc.) according to the manufacturer's protocol. RNA samples were assessed by their optical density at $260 \mathrm{~nm}$, and then reverse transcribed into cDNA using the RevertAid Premium First Strand cDNA Synthesis kit (Fermentas; Thermo Fisher Scientific, Inc, Pittsburgh, PA, USA). RT-qPCR was performed using the SYBR Green PCR Master Mix kit (Applied Biosystems; Thermo Fisher Scientific, Inc.) in conjunction with an ABI Prism 7300 Real-Time PCR System (Applied Biosystems; Thermo Fisher Scientific, Inc.). The TPX2 primers were as follows: Forward, 5'-ACCTTGCCCTACTAAGATT-3' and reverse, 5'-AATGTGGCACAGGTTGAGC-3'. The $\beta$-actin primers were as follows: Forward, 5'-GGGAAATCGTGCGTG
ACAT-3' and reverse, 5'-CTGGAAGGTGGACAGCGAG-3'. The cycling conditions were as follows: $95^{\circ} \mathrm{C}$ for $10 \mathrm{~min}$, followed by 40 cycles at $95^{\circ} \mathrm{C}$ for $15 \mathrm{sec}, 60^{\circ} \mathrm{C}$ for $15 \mathrm{sec}$ and $95^{\circ} \mathrm{C}$ for $15 \mathrm{sec}$. The relative fold-change in expression of mRNAs was calculated using the $2^{-\Delta \Delta \mathrm{Cq}}$ method (13). Each sample was analyzed in triplicate.

Western blotting. Total protein cell lysates were prepared by lysing the cells in radioimmunoprecipitation assay buffer. Protein concentrations were determined using the Pierce BCA Protein Assay kit (Thermo Fisher Scientific, Inc.). In total, $40 \mu \mathrm{g}$ protein from each sample were resolved by $10 \%$ SDS-PAGE and transferred to polyvinylidene difluoride membranes (Merck Millipore, Darmstadt, Germany). Membranes were blocked for $1 \mathrm{~h}$ at room temperature with $5 \%$ non-fat dry milk, followed by incubation overnight at $4{ }^{\circ} \mathrm{C}$ with the following primary antibodies: Rabbit anti-human TPX2 polyclonal antibody (1:200; sc-32863), rabbit anti-human AKT polyclonal antibody (1:200; sc-8312), rabbit anti-human phosphorylated AKT polyclonal antibody (1:200; sc-293095), rabbit anti-MMP-9 polyclonal antibody (1:200; sc-10737), polyclonal mouse anti-human cyclin D1 (1:200; sc-29287) and polyclonal mouse anti-human p21 (1:200; sc-44271). Horseradish peroxidase-conjugated monoclonal goat anti-rabbit or anti-mouse immunoglobulin $\mathrm{G}$ secondary antibodies (1:1,000; W10804 and W10815, respectively; Zymed; Thermo Fisher Scientific, Inc.) and enhanced chemiluminescence were used for detection. Next, membranes were stripped and re-probed with a primary antibody against GAPDH (1:1,000; AP0063; Bioworld Technology, Inc., St. Louis Park, MN, USA), which was used for normalization. Signal intensities were determined using ImageJ 1.37 gel analysis software (National Institutes of Health- Bethesda, MD, USA).

Proliferation assays. Cells $\left(5 \times 10^{3}\right.$ cells/well) were plated in 96-well plates and allowed to grow for 24,48 and $72 \mathrm{~h}$ after transfection with the corresponding siRNAs or expression plasmids. Cell proliferation was documented every $24 \mathrm{~h}$ for 4 days using an MTT assay (Sigma-Aldrich; Merck Millipore). Absorbance was measured at $570 \mathrm{~nm}$ using a microplate reader.

Cell cycle assay. At $48 \mathrm{~h}$ post-transfection, cells were harvested by trypsinization, washed three times with ice-cold PBS and fixed with $70 \%$ ethanol overnight at $4^{\circ} \mathrm{C}$. The fixed cells were rehydrated in PBS and subjected to propidium iodide/RNase staining, followed by fluorescence-activated cell sorting analysis (BD Biosciences, San Jose, CA, USA). The percentage of cells in each phase of the cell cycle was estimated using PVElite 2015 software (Intergraph Corporation, Madison, AL, USA).

Transwell migration assay. Cell invasion was determined using Transwell assays. Briefly, TPX2-overexpressing plasmid and siRNA to knockdown TPX2 expression were transfected into cells, and $48 \mathrm{~h}$ later, $3 \times 10^{4}$ cells were transferred to the top of Matrigel-coated invasion chambers (BD Biosciences) in serum-free DMEM. Next, DMEM containing 10\% FBS was added to the lower chamber, and $24 \mathrm{~h}$ later, the non-invading cells were removed, while the invading cells were fixed with $95 \%$ ethanol and stained with $0.1 \%$ crystal violet. Images were captured in a light microscope under x100 magnification. Experiments were performed three independent times. 
Statistical analysis. All data are shown as the mean + standard deviation. All experiments were repeated three times. All statistical analyses were performed with a two-tailed Student's $t$-test using SPSS version 12.0 software (SPSS, Inc., Chicago, IL, USA). $\mathrm{P}<0.05$ was considered to indicate a statistically significant difference.

\section{Results}

TPX2 mRNA and protein expression in glioma cell lines. RT-qPCR was performed to determine the mRNA expression levels of TPX2 in the glioma cell lines U251, U87 and LN229 and in primary NHAs. TPX2 transcript levels were significantly lower in NHA cells compared with those in all the glioma cell lines (all $\mathrm{P}<0.05$; Fig. 1A). The TPX2 protein levels in all the cell lines mentioned above were also examined, and our findings were consistent with those obtained from RT-qPCR. That is, TPX2 protein levels were lower in NHA cells compared with those in glioma cells (U251, 5.67-fold; U87, 5.27-fold; and LN229, 3.76-fold; all P<0.05; Fig. 1B). These results suggest that TPX2 may function as an oncogene in GBM.

TPX2 overexpression promotes cell proliferation and invasion in U251 and U87 cells. The effect of TPX2 overexpression in the glioma cell lines U251 and U87 was examined. Cells were transfected with pcDNA-TPX2 or pcDNA3.1 as control. MTT assay was performed to measure proliferation, while Transwell assay was performed to assess migration. Compared with the controls, TPX 2 overexpression promoted the proliferation of both U251 ( $24 \mathrm{~h}, 0.362 \pm 0.002$, $0.366 \pm 0.008$ and $0.522 \pm 0.005$ for the mock, pcDNA3.1 and pcDNA-TPX2 groups, respectively; 48 h, 0.554 \pm 0.019 , $0.565 \pm 0.027$ and $0.959 \pm 0.023$ for the mock, pcDNA3.1 and pcDNA-TPX2 groups, respectively; and $72 \mathrm{~h}, 0.894 \pm 0.053$, $0.843 \pm 0.062$ and $1.253 \pm 0.172$ for the mock, pcDNA3.1 and pcDNA-TPX2 groups, respectively; $\mathrm{P}<0.05$; Fig. $2 \mathrm{~A})$ and U87 cells $(24 \mathrm{~h}, 0.358 \pm 0.019,0.362 \pm 0.014$ and $0.569 \pm 0.045$ for the mock, pcDNA3.1 and pcDNA-TPX2 groups, respectively; $48 \mathrm{~h}, 0.555 \pm 0.017,0.567 \pm 0.008$ and $0.933 \pm 0.010$ for the mock, pcDNA3.1 and pcDNA-TPX2 groups, respectively; and $72 \mathrm{~h}, 0.790 \pm 0.018,0.798 \pm 0.025$ and $1.241 \pm 0.035$ for the mock, pcDNA3.1 and pcDNA-TPX2 groups, respectively; $\mathrm{P}<0.05$; Fig. $2 \mathrm{~B}$ ). TPX2 overexpression also decreased the percentage of cells in the G0/G1 phase (U251 cells, $34.730 \pm 0.470,35.481 \pm 0.671$ and $21.092 \pm 0.708$ for the mock, pcDNA3.1 and pcDNA-TPX2 groups, respectively; U87 cells, $34.513 \pm 0.438,34.114 \pm 0.909$ and $19.623 \pm 0.578$ for the mock, pcDNA3.1 and pcDNA-TPX2 groups, respectively; $\mathrm{P}<0.05$; Fig. $2 \mathrm{C}$ and $\mathrm{D}$, respectively). Overexpression of TPX2 also increased cellular migration (U251 cells, $105.200 \pm 3.114,100.600 \pm 3.361$ and $153.000 \pm 5.612$ for the mock, pcDNA3.1 and pcDNA-TPX2 groups, respectively; U87 cells, $102.000 \pm 1.870,104.800 \pm 1.303$ and $152.200 \pm 3.114$ for the mock, pcDNA3.1 and pcDNA-TPX2 groups, respectively; $\mathrm{P}<0.05$; Fig. 3). These results suggest that TPX2 may promote cellular proliferation and invasion in glioma cells.

TPX2 overexpression activates AKT signaling in U251 and U87 cells. AKT protein kinases are critical regulators of multiple cellular functions, including cell growth, survival and

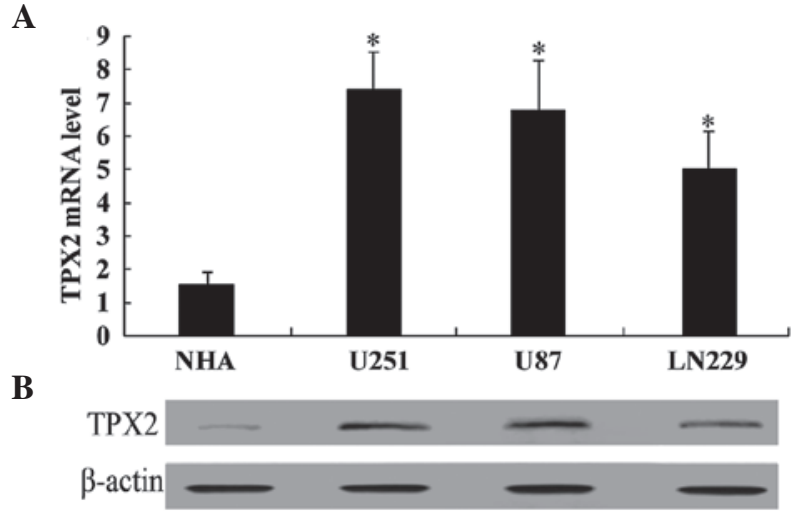

Figure 1. TPX2 expression in primary NHAs and glioma cell lines. (A) TPX2 mRNA expression in primary NHAs and in and glioma cell lines (U251, U87 and LN229) was assessed by RT-qPCR. RT-qPCR revealed that TPX2 was upregulated in all the glioma cell lines compared with the NHA cells. ${ }^{*} \mathrm{P}<0.05$ vs. NHAs. (B) TPX2 protein expression in primary NHAs and glioma cell lines (U251, U87 and LN229) was detected by western blotting. NHA, normal human astrocyte; TPX2, targeting protein for Xenopus kinesin-like protein 2; mRNA, messenger RNA; RT-qPCR, reverse transcription-quantitative polymerase chain reaction.

metabolism (14). Cyclin D1 and p21 are key pro- and anti-cell cycle regulators, respectively. In the present study, TPX2 overexpression significantly increased AKT phosphorylation in U251 and U87 cells ( $\mathrm{P}<0.05$; Fig 4). Additionally, overexpression of TPX2 decreased the levels of p21 and increased the levels of both cyclin D1 and MMP-9 in U251 and U87 glioma cells.

TPX2 knockdown suppresses proliferation and invasion in U251 and U87 cells. The effect of TPX2 knockdown on the proliferation and invasion abilities of the U251 and U87 glioma cell lines was next examined. The results from MTT assay revealed that TPX2 knockdown decreased the cellular proliferation of both U251 ( $24 \mathrm{~h}, 0.386 \pm 0.006,0.379 \pm 0.002$ and $0.228 \pm 0.008$ for the mock, pcDNA3.1 and pcDNA-TPX2 groups, respectively; $48 \mathrm{~h}, 0.579 \pm 0.013,0.587 \pm 0.007$ and $0.327 \pm 0.009$ for the mock, pcDNA3.1 and pcDNA-TPX2 groups, respectively; and $72 \mathrm{~h}, 0.756 \pm 0.015,0.747 \pm 0.039$ and $0.517 \pm 0.008$ for the mock, pcDNA3.1 and pcDNA-TPX2 groups, respectively; $\mathrm{P}<0.05$; Fig. 5A) and U87 cells ( $24 \mathrm{~h}, 0.368 \pm 0.009$, $0.376 \pm 0.005$ and $0.223 \pm 0.006$ for the mock, pcDNA3.1 and pcDNA-TPX2 groups, respectively; $48 \mathrm{~h}, 0.577 \pm 0.009$, $0.585 \pm 0.008$ and $0.367 \pm 0.007$ for the mock, pcDNA3.1 and pcDNA-TPX2 groups, respectively; and $72 \mathrm{~h}, 0.814 \pm 0.012$, $0.777 \pm 0.009$ and $0.585 \pm 0.047$ for the mock, pcDNA3.1 and pcDNA-TPX2 groups, respectively; $\mathrm{P}<0.05$; Fig. 5B) compared with the mock control group and the control siRNA group. Consistent with this, cell cycle analysis revealed that TPX2 knockdown increased the percentage of cells in G0/G1 phase and decreased the percentage of cells in S phase (U251 cells, $25.720 \pm 0.991,27.246 \pm 0.503$ and $45.034 \pm 4.496$ for the mock, pcDNA3.1 and pcDNA-TPX2 groups, respectively; Fig. 5C; U87 cells, $28.558 \pm 0.528,29.174 \pm 0.578$ and $41.006 \pm 1.158$ for the mock, pcDNA3.1 and pcDNA-TPX2 groups, respectively; $\mathrm{P}<0.05$; Fig. 5D). In addition to decreasing proliferation, TPX2 knockdown also reduced the ability of U251 (108.2 \pm 2.863 , $105.2 \pm 1.303$ and $55.6 \pm 2.073$ for the mock, pcDNA3.1 and pcDNA-TPX2 groups, respectively; P<0.05; Fig. 6) and U87 
A

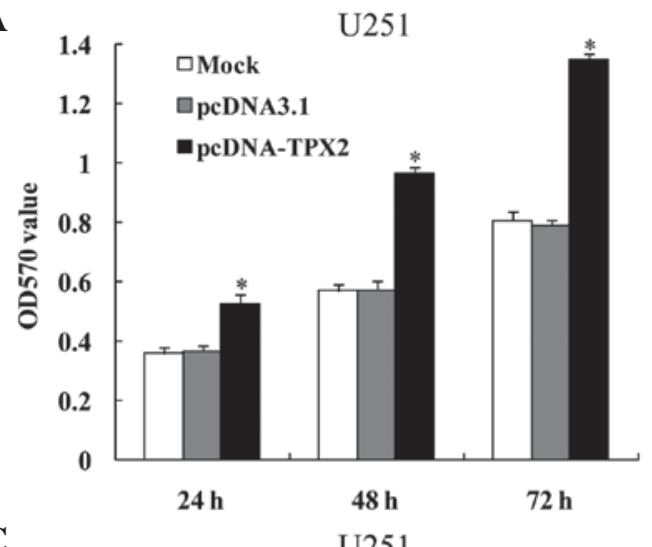

C

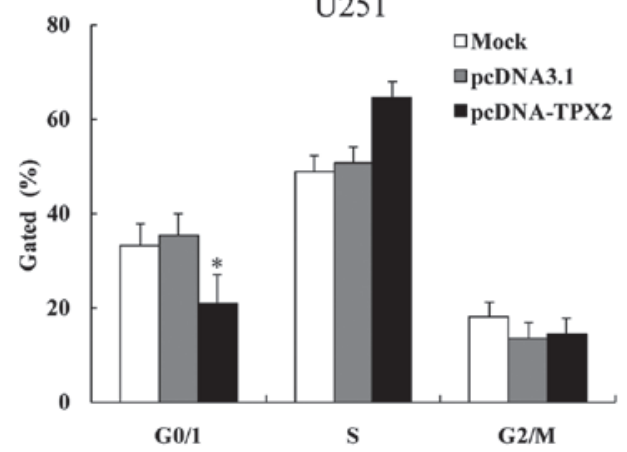

B
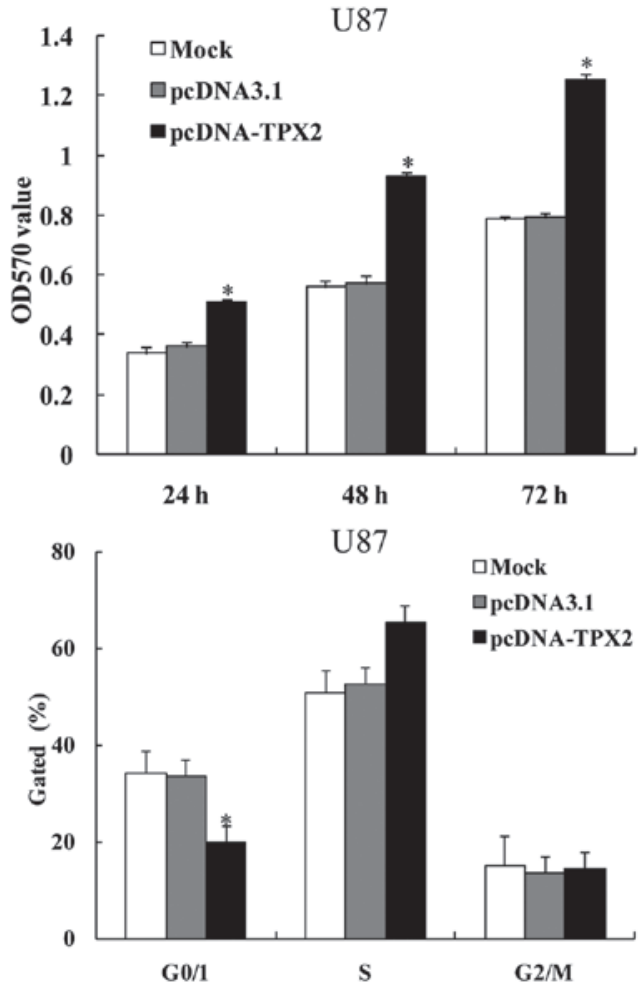

Figure 2. TPX2 overexpression promotes cell proliferation in U251 and U87 cells. (A and B) TPX2 overexpression significantly promotes the proliferation of (A) U251 and (B) U87 glioma cells at different time points. (C and D) TPX2 overexpression led to a reduced number of (C) U251 and (D) U87 glioma cells in G0/G1 phase and an increased number of cells in $\mathrm{S}$ phase. Data are presented as the mean +standard deviation of three independent experiments. "P<0.05. TPX2, targeting protein for Xenopus kinesin-like protein 2; OD, optical density.

A

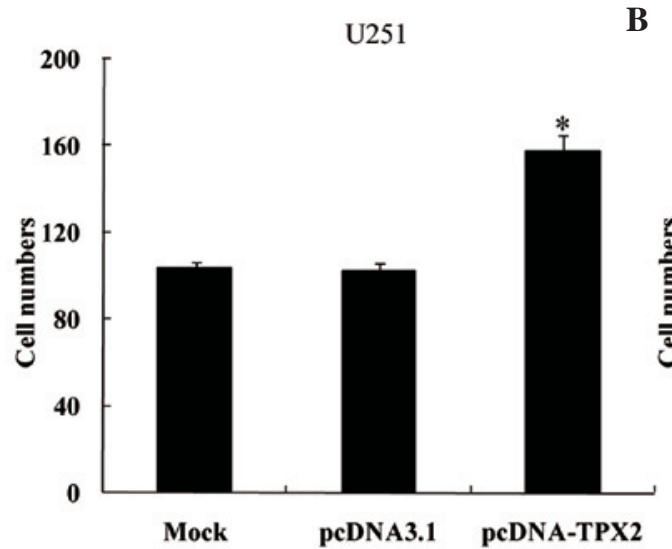

C

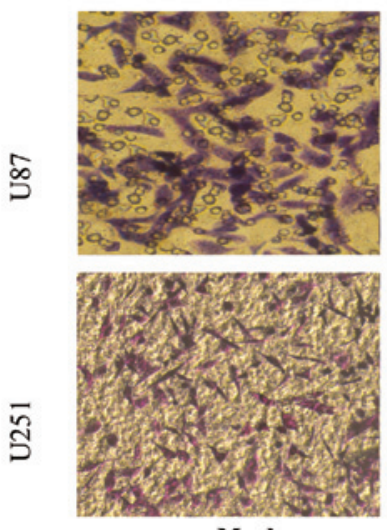

Mock

B

peDNA3.1
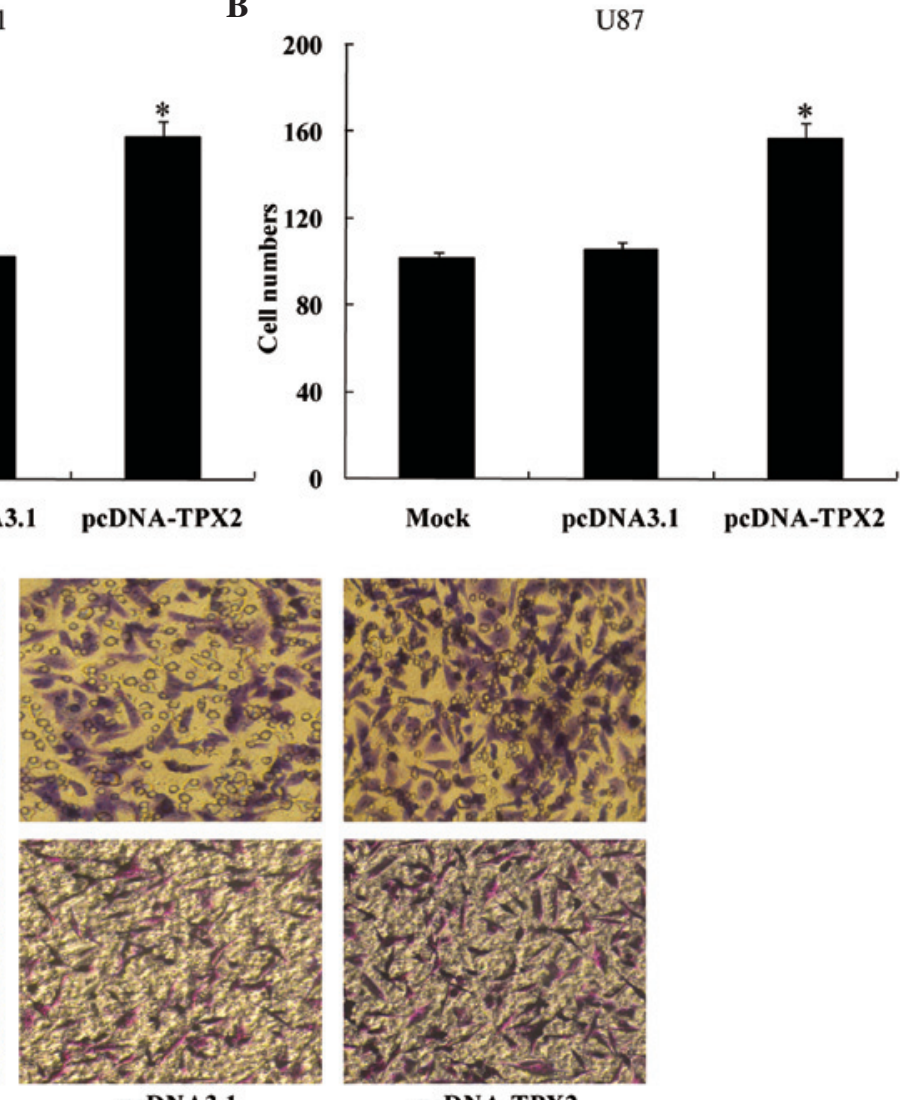

pCDNA-TPX2

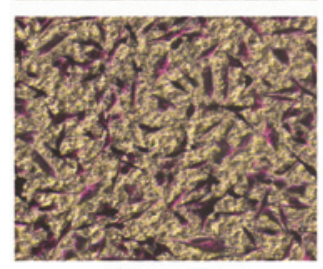

Figure 3. TPX2 overexpression promotes invasion in U251 and U87 cells. (A) U251 and (B) U87 cells were examined for cell invasion in 24-well plates with Transwell chambers. (C) The migrated cells were stained with crystal violet and observed under a light microscope (magnification, x200). The invasiveness of U251 and U87 cells was enhanced with TPX2 overexpression. " $\mathrm{P}<0.05$. TPX2, targeting protein for Xenopus kinesin-like protein 2. 

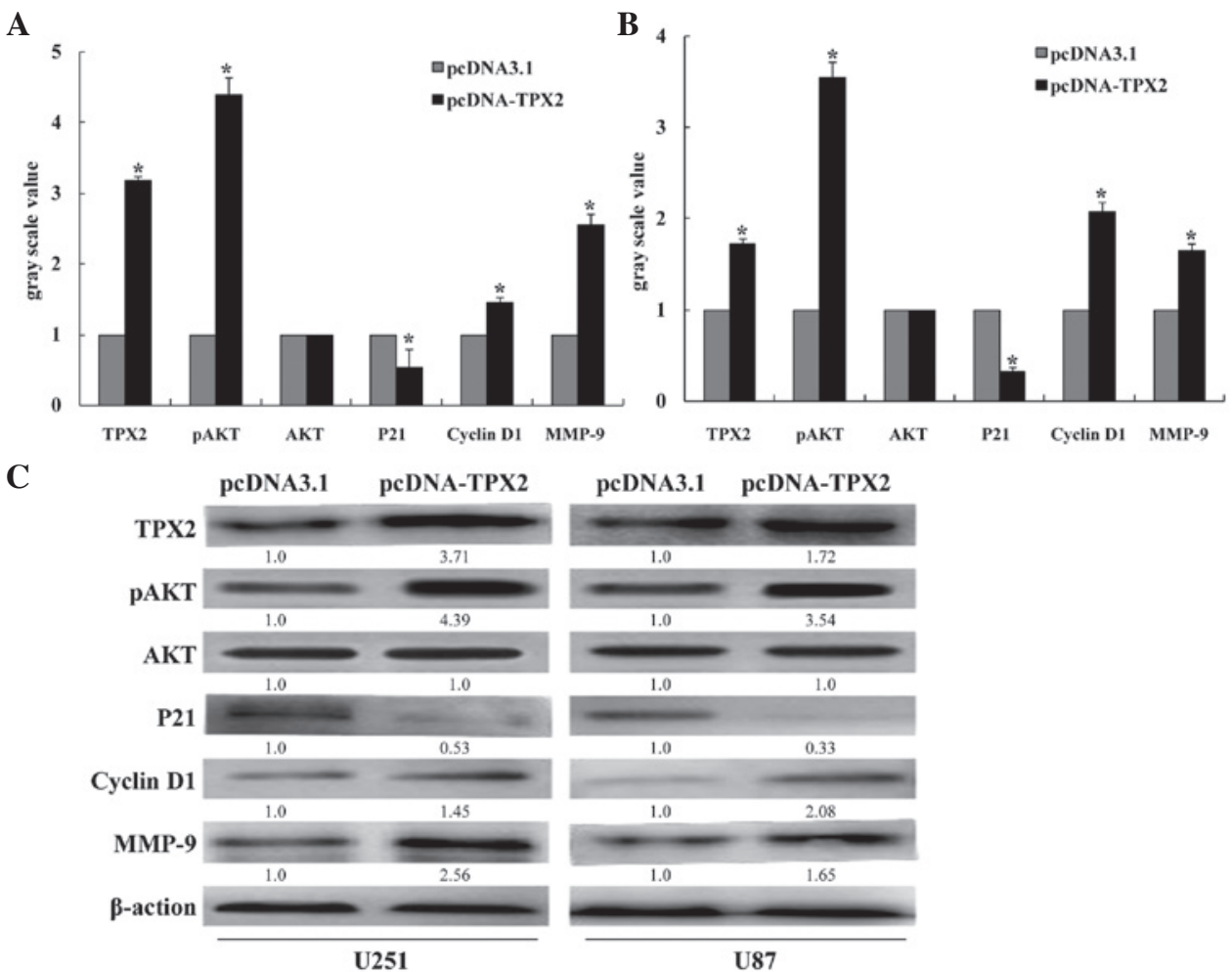

Figure 4. TPX2 overexpression activates AKT signaling pathway in (A) U251 and (B) U87 cells. (C) Western blot analysis of TPX2, pAKT, AKT, p21, cyclin D1 and MMP-9 expression in cells transfected with pcDNA-TPX2 or pcDNA3.1. $\beta$-actin served as the loading control ("P<0.05). TPX2, targeting protein for Xenopus kinesin-like protein 2; MMP, matrix metallopeptidase; p, phosphorylated.

A

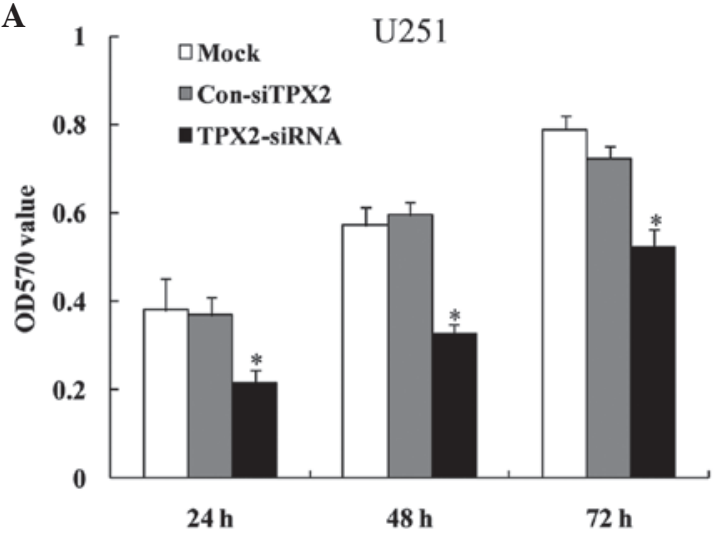

C

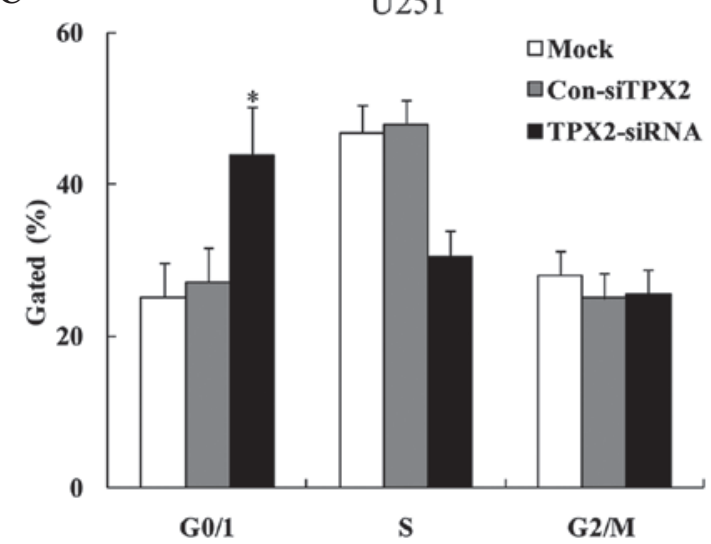

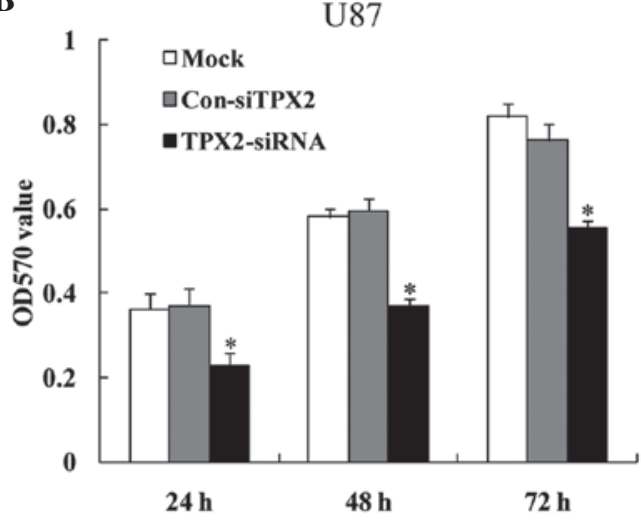

D

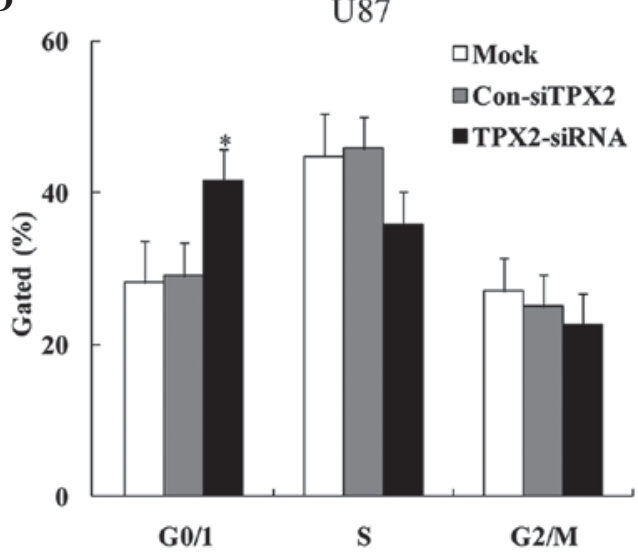

Figure 5. TPX2 knockdown suppresses cell proliferation in U251 and U87 cells. (A and B) TPX2 knockdown significantly suppressed the proliferation of (A) U251 and (B) U87 glioma cells at different time points. (C and D) TPX2 knockdown resulted in an increased number of (C) U251 and (D) U87 glioma cells in G0/G1 phase and a reduced number of cells in S phase. Data are the mean + standard deviation of three independent experiments. "P<0.05. TPX2, targeting protein for Xenopus kinesin-like protein 2; Con, control; siRNA, small interfering RNA; OD, optical density. 


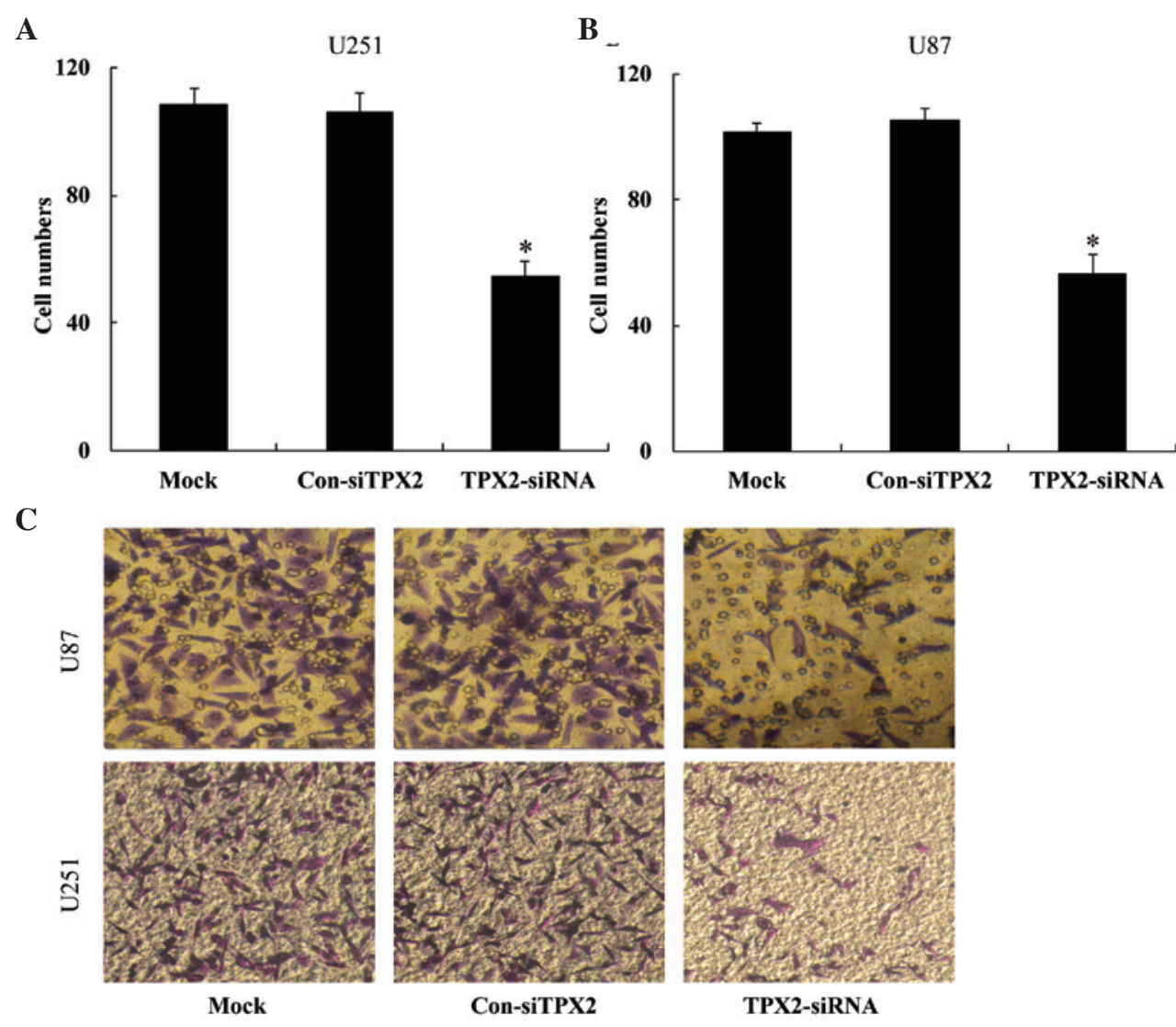

Figure 6. TPX2 knockdown suppresses the invasion capacity of (A) U251 and (B) U87 cells. (A-C) U251 and U87 cells were examined for cell invasion in 24-well plates with Transwell chambers. (C) The migrated cells were stained with crystal violet and observed under a light microscope (magnification, x200). The invasiveness of U251 and U87 cells was attenuated with TPX2 knockdown. "P<0.05. TPX2, targeting protein for Xenopus kinesin-like protein 2; Con, control; siRNA, small interfering RNA.

cells $(103.0 \pm 2.121,105.6 \pm 1.517$ and $55.6 \pm 2.073$ for the mock, pcDNA3.1 and pcDNA-TPX2 groups, respectively; $\mathrm{P}<0.05$; Fig. 6) to invade the Transwell chamber. Taken together, these findings suggest that TPX2 knockdown suppresses the proliferation and invasion capacities of glioma cells.

TPX2 knockdown inhibits AKT signaling in U251 and U87 cells. The effect of TPX2 knockdown on AKT signaling in various glioma cell lines was next examined. Compared with control siRNA-transfected cells, TPX2 knockdown significantly decreased the phosphorylation of AKT. Additionally, knockdown of TPX2 increased the expression of the cell cycle inhibitor p21. By contrast, cyclin D1 and MMP-9 were downregulated in U251 and U87 glioma cells ( $<<0.05$; Fig. 7) following transfection with TPX2 siRNA. It was also observed that treatment of cells with AKT inhibitor IV in large part phenocopied the effect of TPX2 knockdown. That is, AKT inhibitor IV decreased the phosphorylation of AKT, decreased the expression of MMP-9 and cyclin D1, and increased the expression of p21 in U251 and U87 cells $(\mathrm{P}<0.05$; Fig. 8). These results suggest that TPX2-mediated control of glioma cell proliferation and invasion may occur via the AKT signaling pathway.

\section{Discussion}

GBM is the most common type of human brain tumor and is essentially incurable. Despite recent advances in GBM treatment, including the use of aggressive surgery combined with radiation, chemotherapy and biological therapy, the median survival values have not changed much over the past several years $(15,16)$. Thus, improved detection, prevention and treatment of GBM are important unmet needs. Invasive growth is the most characteristic phenotype of glioblastoma, which is a major reason why patients with this type of tumor have such poor prognosis (2). Therefore, inhibition of invasion, for example by interfering with the signaling pathways that regulate this process, may be therapeutically useful for the treatment of GBM.

TPX2 regulates multiple aspects of mitotic spindle assembly and chromosome isolation $(5,17)$. A previous study revealed that TPX2 overexpression promotes invasion and progression in vitro (18), and suggested that TPX2 is a potent oncogene in human cancers (6-9). Li et al (9) reported that TPX2 expression was elevated in glioma tissue compared with normal tissue. TPX2 knockdown has been shown to inhibit cell proliferation and to increase early-stage apoptosis in glioma U87 cells (9). Knockdown of TPX2 has also been shown to decrease the levels of Aurora A, Ran and cyclin B1, and to increase the expression of c-Myc and p53 (9). Despite these findings, the precise role of TPX2 in glioma is poorly understood.

The present study demonstrates that TPX2 expression is significantly higher in several glioma cell lines compared with that in NHAs. It also provides data showing that TPX2 regulates the proliferation and invasion of abilities U251 and 

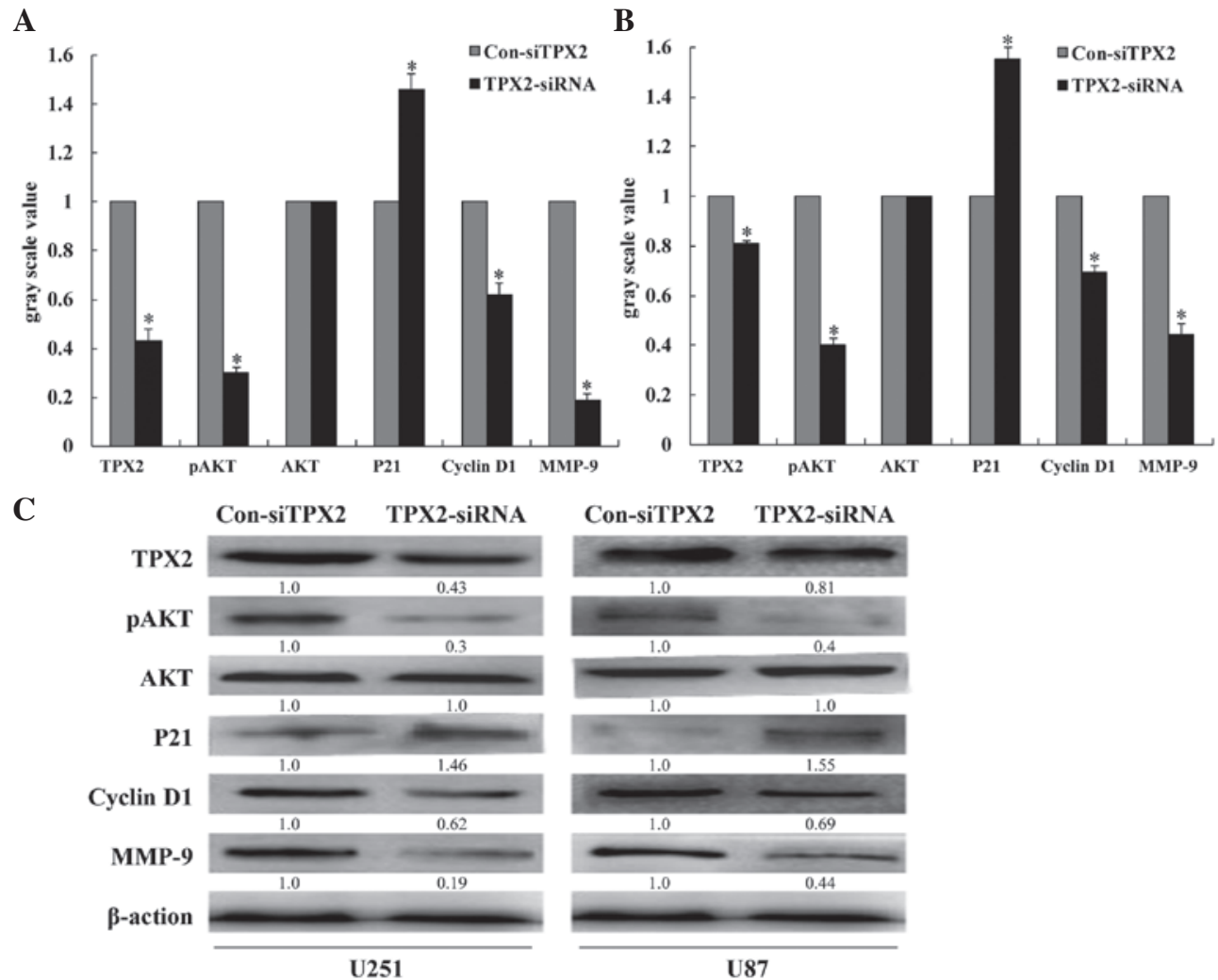

Figure 7. TPX2 knockdown inhibits AKT signaling pathway in (A) U251 and (B) U87 cells. (C) Western blot analysis of TPX2, pAKT, AKT, p21, cyclin D1 and MMP-9 expression in cells transfected with TPX2-siRNA or Con-siRNA. $\beta$-actin served as the loading control ("P<0.05). TPX2, targeting protein for Xenopus kinesin-like protein 2; MMP, matrix metallopeptidase; p, phosphorylated; Con, control; siRNA, small interfering RNA.
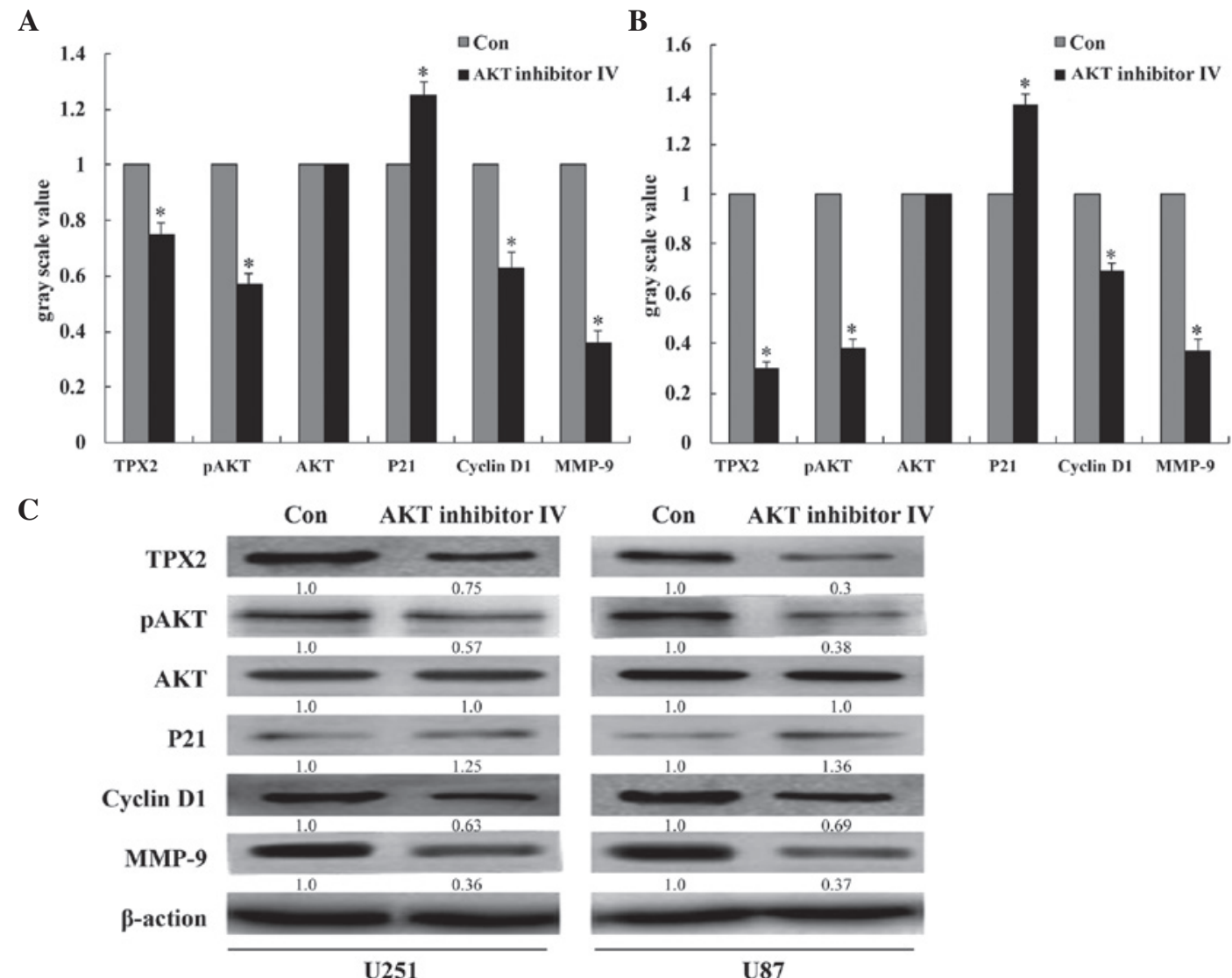

U251

U87

Figure 8. AKT inhibitor IV inhibits TPX2 expression and AKT signaling pathway in (A) U251 and (B) U87 cells. (C) Western blot analysis of TPX2, pAKT, AKT, p21, cyclin D1 and MMP-9 expression following AKT inhibitor IV treatment. $\beta$-actin served as the loading control ("P<0.05). TPX2, targeting protein for Xenopus kinesin-like protein 2; MMP, matrix metallopeptidase; p, phosphorylated; Con, control; siRNA, small interfering RNA. 
U87 cells. Specifically, overexpression of TPX2 in either cell line promoted cell proliferation and enhanced their invasive potential. Importantly, knockdown of TPX2 had the exact opposite effect. Taken together, these data suggest that TPX2 regulates cell proliferation and invasion in glioma cells.

Previous in vivo and in vitro glioma studies noticed that AKT activation and phosphorylation are common occurrences in $\operatorname{GBM}(19,20)$. AKT protein kinases are critical regulators of multiple cellular functions, including cell growth, survival and metabolism (14). In normal cells, the growth factor-dependent activation of phosphoinositide 3-kinase (PI3K) is tightly controlled by the potent tumor suppressor phosphatase and tensin homolog (PTEN). Aberrant activation of AKT can result from mutations in upstream regulators (such as receptor tyrosine kinases), overexpression of AKT or deletions of negative regulators (such as PTEN) (21). AKT signaling regulates cell cycle entry by phosphorylating cyclin D1-cyclin-dependent kinase 4 complexes $(22,23)$. AKT promotes the G1/S transition by antagonizing the accumulation of two G1/S transition inhibitors, p21 and p27 (24). p21 can be phosphorylated either directly by AKT or by kinases activated by AKT (25). MMP-2 and MMP-9 are key enzymes involved in the degradation of type IV collagen, which is a component of the extracellular matrix. Increased expression of both MMP-2 and MMP-9 has been associated with tumor cell growth and invasion (26). Fu et al demonstrated that activation of PI3K/AKT signaling suppressed the expression of p53 and tissue inhibitor of MMP-2, resulting in overexpression of proliferating cell nuclear antigen, cyclin D1, MMP-2 and MMP-9, which promoted cell proliferation and invasion in U251 glioma cells (27).

The data presented in the current study demonstrate that TPX2 significantly increases AKT phosphorylation in U251 and U87 glioma cells. This is associated with altered levels of cell cycle regulators such as cyclin D1 and p21. It was also observed that TPX2 regulates the levels of MMP-9, which may be associated with the invasive potential of glioma cells. Taken together, our results demonstrate that TPX2 promotes proliferation and invasion via activating AKT signaling in glioma cells. Additionally, these findings suggest that TPX2 may be a potential therapeutic target in glioblastoma.

\section{References}

1. Stupp R, Mason WP, van den Bent MJ, Weller M, Fisher B, Taphoorn MJ, Belanger K, Brandes AA, Marosi C, Bogdahn U, et al: Radiotherapy plus concomitant and adjuvant temozolomide for glioblastoma. N Engl J Med 352: 987-996, 2005.

2. Wu DG, Wang YY, Fan LG, Luo H, Han B, Sun LH, Wang XF, Zhang JX, Cao L, Wang XR, et al: MicroRNA-7 regulates glioblastoma cell invasion via targeting focal adhesion kinase expression. Chin Med J (Engl) 124: 2616-2621, 2011.

3. Heidebrecht HJ, Buck F, Steinmann J, Sprenger R, Wacker HH and Parwaresch R: p100: A novel proliferation-associated nuclear protein specifically restricted to cell cycle phases $\mathrm{S}, \mathrm{G} 2$, and $\mathrm{M}$. Blood 90: 226-233, 1997.

4. Kufer TA, Silljé HH, Körner R, Gruss OJ, Meraldi P and Nigg EA: Human TPX2 is required for targeting Aurora-A kinase to the spindle. J Cell Biol 158: 617-623, 2002.

5. Gruss OJ, Wittmann M, Yokoyama H, Pepperkok R, Kufer T, Silljé H, Karsenti E, Mattaj IW and Vernos I: Chromosome-induced microtubule assembly mediated by TPX2 is required for spindle formation in HeLa cells. Nat Cell Biol 4: 871-879, 2002.
6. Li Y, Tang H, Sun Z, Bungum AO, Edell ES, Lingle WL, Stoddard SM, Zhang M, Jen J, Yang P and Wang L: Network-based approach identified cell cycle genes as predictor of overall survival in lung adenocarcinoma patients. Lung Cancer 80: 91-98, 2013.

7. Scotto L, Narayan G, Nandula SV, Arias-Pulido H, Subramaniyam S, Schneider A, Kaufmann AM, Wright JD, Pothuri B, Mansukhani M and Murty VV: Identification of copy number gain and overexpressed genes on chromosome arm $20 \mathrm{q}$ by an integrative genomic approach in cervical cancer: Potential role in progression. Genes Chromosomes Cancer 47: 755-765, 2008.

8. Yan L, Li S, Xu C, Zhao X, Hao B, Li H and Qiao B: Target protein for Xklp2 (TPX2), a microtubule-related protein, contributes to malignant phenotype in bladder carcinoma. Tumour Biol 34: 4089-4100, 2013.

9. Li B, Qi XQ, Chen X, Huang X, Liu GY, Chen HR, Huang CG, Luo $\mathrm{C}$ and Lu YC: Expression of targeting protein for Xenopus kinesin-like protein 2 is associated with progression of human malignant astrocytoma. Brain Res 1352: 200-207, 2010.

10. Gao J, Ding F, Liu Q and Yao Y: Knockdown of MACC1 expression suppressed hepatocellular carcinoma cell migration and invasion and inhibited expression of MMP-2 and MMP-9. Mol Cell Biochem 376: 21-32, 2013.

11. Wang YH, Dong YY, Wang WM, Xie XY, Wang ZM, Chen RX, Chen J, Gao DM, Cui JF and Ren ZG: Vascular endothelial cells facilitated HCC invasion and metastasis through the Akt and $\mathrm{NF}-\kappa \mathrm{B}$ pathways induced by paracrine cytokines. J Exp Clin Cancer Res 32: 51, 2013.

12. Liu Q, Yang P, Tu K, Zhang H, Zheng X, Yao Y and Liu Q: TPX2 knockdown suppressed hepatocellular carcinoma cell invasion via inactivating AKT signaling and inhibiting MMP-2 and MMP-9 expression. Chin J Cancer Res 26: 410-417, 2014

13. Livak KJ and Schmittgen TD: Analysis of relative gene expression data using real-time quantitative PCR and the 2(-Delta Delta C(T)) Method. Methods 25: 402-408, 2001.

14. Vivanco I and Sawyers CL: The phosphatidylinositol 3-kinase AKT pathway in human cancer. Nat Rev Cancer 2: 489-501, 2002.

15. Clarke J, Butowski N and Chang S: Recent advances in therapy for glioblastoma. Arch Neurol 67: 279-283, 2010.

16. Liu R, Page M, Solheim K, Fox S and Chang SM: Quality of life in adults with brain tumors: Current knowledge and future directions. Neuro Oncol 11: 330-339, 2009.

17. Neumayer G, Belzil C, Gruss OJ and Nguyen MD: TPX2: Of spindle assembly, DNA damage response, and cancer. Cell Mol Life Sci 71: 3027-3047, 2014.

18. Chang H, Wang J, Tian Y, Xu J, Gou X and Cheng J: The TPX2 gene is a promising diagnostic and therapeutic target for cervical cancer. Oncol Rep 27: 1353-1359, 2012.

19. Choe G, Horvath S, Cloughesy TF, Crosby K, Seligson D, Palotie A, Inge L, Smith BL, Sawyers CL and Mischel PS: Analysis of the phosphatidylinositol3'-kinase signaling pathway in glioma patients in vivo. Cancer Res 63: 2742-2746, 2003.

20. Haas-Kogan D, Shalev N, Wong M, Mills G, Yount G and Stokoe D: Protein kinase B (PKB/Akt) activity is elevated in glioma cells due to mutation of the tumor suppressor PTEN/MMAC. Curr Biol 8: 1195-1198, 1998.

21. Robinson GL, Robinson JP, Lastwika KJ, Holmen SL and Vanbrocklin MW: Akt signaling accelerates tumor recurrence following ras inhibitionin the context of ink4a/arf loss. Genes Cancer 4: 476-485, 2013.

22. Diehl JA, Cheng M, Roussel MF and Sherr CJ: Glycogen synthase kinase-3beta regulates cyclin D1 proteolysis and subcellular localization. Genes Dev 12: 3499-3511, 1998.

23. Cross DA, Alessi DR, Cohen P, Andjelkovich M and Hemmings BA: Inhibition of glycogen synthase kinase -3 by insulin mediated by protein kinase B. Nature 378: 785-789, 1995.

24. Lee J and Kim SS: The function of p27 KIP1 during tumor development. Exp Mol Med 41: 765-771, 2009.

25. Child ES and Mann DJ: The intricacies of p21 phosphorylation: Protein/protein interactions, subcellular localization and stability. Cell Cycle 5: 1313-1319, 2006.

26. Cawston TE and Wilson AJ: Understanding the role of tissue degrading enzymes and their inhibitors in development and disease. Best Pract Res Clin Rheumatol 20: 983-1002, 2006.

27. Fu Y, Zhang Q, Kang C, Zhang J, Zhang K, Pu P, Wang G and Wang T: Inhibitory effects of adenovirus mediated Akt1 and PIK3R1 shRNA on the growth of malignant tumor cells in vitro and in vivo. Cancer Biol Ther 8: 1002-1009, 2009. 